\title{
Neutrophils deliver the goods
}

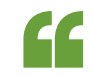

The authors decided to of these properties and test the potential of drug carriers take advantage neutrophils as 5

Glioblastoma is a notoriously difficult cancer to treat, partly because its invasive nature makes surgery challenging, and partly because the blood-brain barrier (BBB) impedes the delivery of chemotherapeutics. Delivery of therapeutic agents using endogenous carrier cells is a promising strategy for the treatment of brain tumours, as these cells can traverse the $\mathrm{BBB}$, infiltrate the tumour and recruit additional circulating carrier cells. Now, reporting in Nature Nanotechnology, the team led by Mo and Zhang have shown that neutrophils carrying liposomes that contain the antimitotic drug paclitaxel (PTX) can penetrate the brain and suppress the recurrence of glioma in mice, thereby significantly improving survival.

Neutrophils can be recruited to the tumour site in response to chemokines released after surgical tumour removal. The authors decided to take

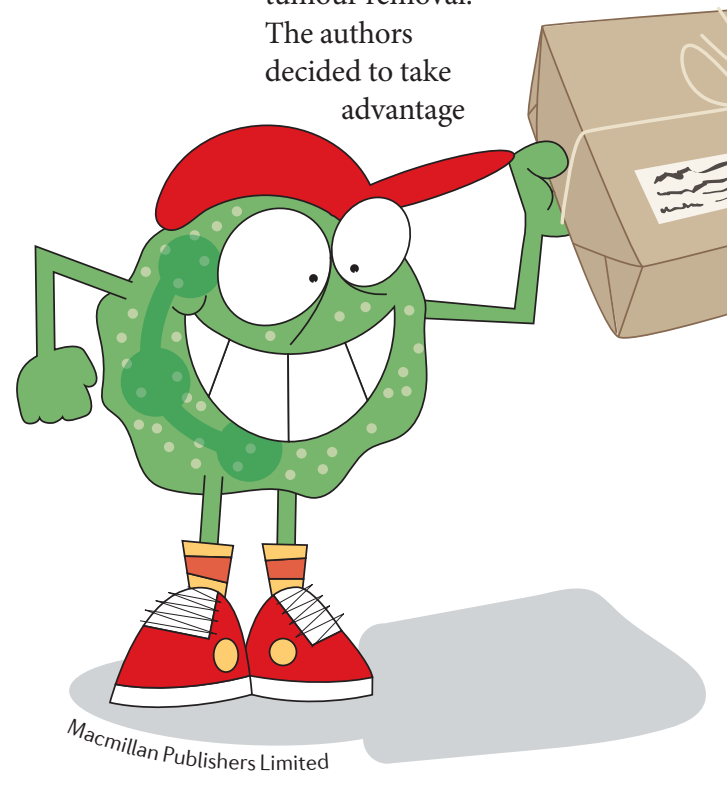

of these properties and test the potential of neutrophils as drug carriers. They isolated and purified mature neutrophils from the mouse bone marrow and incubated them with PTX encapsulated in cationic liposomes (PTX-CL) to protect the carrier cells from the effects of the drug. Indeed, neutrophils loaded with PTX-CL were not affected by the drug at any of the doses tested and retained their normal morphology and physiological activities, migrating in response to inflammatory stimuli.

The authors then tested the stability of PTX-CL and its release from neutrophils, as well as the migratory and cytotoxic capabilities of the loaded neutrophils, in vitro. At physiological conditions, PTX was maintained for 8 hours but released after 4 hours of incubation with phorbol 12-myristate 13-acetate (PMA), which activates protein kinase $\mathrm{C}$

(PKC)-mediated degranulation. Most of the PTX $(88 \%)$ released from neutrophils was encapsulated in the liposomes.

PTX-CL-containing neutrophils migrated efficiently across a murine-brain microvascular endothelial cell (bEnd.3) monolayer - an in vitro model of the BBB - in response to $\mathrm{N}$-formylmethionylleucylphenylalanine (fMLP), a neutrophil chemotactic peptide. PTX-CL neutrophils were able to penetrate the tumour in a 3D multicellular tumour spheroid model. Finally, treatment with PTX-CL-loaded neutrophils induced cytotoxicity in a mouse glioma cell line.

To test for in vivo efficacy, the team intravenously injected the PTX-CL-loaded neutrophils in mice that had undergone surgical resection of an in situ glioma generated by intracranial injection of glioma cells. The neutrophils migrated to the surgical margin and were activated by signals in the inflamed brain. This activation led to subsequent disruption and release of PTX-CL. The 50\% survival rate in mice treated with PTX-CL-loaded neutrophils was up to 61 days compared with 29 days in mice treated with PTX and 38 days in mice treated with PTX-CL. However, the neutrophil-treated mice were not cured, which might be because neutrophils do not reach all the infiltrated tumour cells beyond the surgical margin. Treatment of mice that did not undergo surgery led to limited neutrophil recruitment to the tumour and failed to extend survival, which confirmed that the therapeutic effect depends on the inflammation triggered after surgery.

These results highlight the potential of a neutrophil-based drug delivery system for the treatment of brain tumours and potentially other conditions that involve neutrophil infiltration.

M. Teresa Villanueva

ORIGINAL ARTICLE Xue, J. et al. Neutrophilmediated anticancer drug delivery for suppression of postoperative malignant glioma recurrence. Nat. Nanotech. 12, 692-700 (2017) 